\title{
Administration of Antithrombotic Treatment Was a Risk Factor for Intraperitoneal Hemorrhage in Patients Undergoing Percutaneous Interventions for Liver Diseases.
}

\author{
Akira Asai ( $\square$ in2108@osaka-med.ac.jp ) \\ 2nd department of internal medicine, Osaka Medical College \\ Keisuke Yokohama \\ 2nd department of internal medicine, Osaka Medical College \\ Hideko Ohama \\ 2nd department of internal medicine, Osaka Medical College \\ Yusuke Tsuchimoto \\ 2nd department of internal medicine, Osaka Medical College \\ Shinya Fukunishi \\ 2nd department of internal medicine, Osaka Medical College \\ Kazuhide Higuchi \\ 2nd department of internal medicine, Osaka Medical College
}

\section{Research Article}

Keywords: Percutaneous, diseases, hemorrhage

Posted Date: January 27th, 2021

DOI: https://doi.org/10.21203/rs.3.rs-150259/v1

License: (a) (i) This work is licensed under a Creative Commons Attribution 4.0 International License.

Read Full License 


\section{Abstract}

Percutaneous interventions for liver diseases are currently essential for diagnosis and treatment. The most frequent complication of percutaneous interventions is intraperitoneal hemorrhage. Recently, the number of patients with liver diseases and administered antithrombotics has been increasing. This retrospective cohort study aimed to evaluate the risk factors for intraperitoneal hemorrhage after percutaneous interventions for liver diseases. This study recruited 1025 patients who underwent percutaneous interventions for liver diseases from April 2015 to March 2020. All interventions were performed using an ultrasound-guided approach. Intraperitoneal hemorrhage after percutaneous interventions was detected by abdominal computed tomography. Intraperitoneal hemorrhage occurred in 9 patients $(0.88 \%)$, and these adverse events weren't severe. We compared clinical characteristics between the patients with intraperitoneal hemorrhage and without. There were differences regarding patients who underwent percutaneous treatments of liver tumor $(p=0.1271)$, administration of antithrombotics $(p=0.1961)$, and prothrombin time $(p=0.1683)$. Thereafter, to evaluate the influence of antithrombotics on hemorrhage, we compared the patients with antithrombotics and without. After propensity score matching, hemorrhage in patients treated with antithrombotics was significantly increased than in those without $(p=0.0407)$. The administration of antithrombotics was a risk factor for intraperitoneal hemorrhage in patients undergoing percutaneous interventions for liver diseases.

\section{Introduction}

Percutaneous interventions for liver diseases are currently essential for both diagnosis and treatment ${ }^{1-3}$. Although, these interventions were previously performed blindly or guided by computed tomography (CT), recently, most of them have been ultrasound-guided ${ }^{4}$. Percutaneous interventions for diagnosis are mostly performed by liver biopsy. There are globally 123 million prevalent cases of cirrhosis in 2017 worldwide ${ }^{5}$. Liver biopsy is performed for histopathological interpretation when information for diagnosis, management, or prognostication is not available from non-invasive techniques. Percutaneous interventions for the treatment of liver diseases are mainly performed for liver tumors, including hepatocellular carcinoma and metastatic liver tumor. In addition, radio frequency ablation (RFA) and microwave ablation (MWA) are used in these treatments. These are treatments that use ultrasound image guidance to place a needle through the skin into liver tumors. Worldwide, liver cancer was the fifth most common cancer in 2017, with an estimated 953,000 new cases ${ }^{6}$. However, complications of percutaneous interventions such as hemorrhage, liver abscess, hepatic injury, extrahepatic organ injury, tumor progression, and thermal injuries to the skin have been reported. Moreover, the most frequent complication of these interventions is intraperitoneal bleeding $(0.2 \%)^{7-9}$. In most cases of intraperitoneal hemorrhage, it is possible to be controlled by observation and hemostatic agents. However, some cases have been reported to require blood transfusion or become fatal ${ }^{10,11}$.

Recently, patients with chronic liver diseases who were administered antithrombotic drugs such as antiplatelet and anticoagulant drugs have been increasing ${ }^{12}$. There are two reasons why: these patients 
are aging and sometimes have multiorgan diseases ${ }^{13}$. The other reason might be that the ratio of nonalcoholic steatohepatitis (NASH) among other causes of chronic liver diseases in these patients is increasing ${ }^{14}$. NASH is associated with extrahepatic manifestations such as cardiovascular disease, hypertension, and hyperlipidemia ${ }^{15,16}$. Therefore, these patients need to be administered single or dual antithrombotic therapy. However, in many patients with chronic liver disease, there are inherently abnormalities in hematological parameters, with disturbances in both thrombolysis and coagulation. Usually, we performed interventions according to the guidelines for liver biopsy ${ }^{17}$. In these guidelines for liver biopsy, discontinuation of antithrombotic therapy is recommended for patients before interventions. However, this recommendation is based on other guidelines for perioperative management of anticoagulation and antiplatelet therapy from the British Society for Hematology ${ }^{18}$. Moreover, there are no specific recommendations for patients requiring liver biopsy in these guidelines by the British society of gastroenterology (BSG), and it is stated that the evidence is weak. Furthermore, there is no guideline for percutaneous treatments of liver tumors. In addition, no recommendations for using antithrombotic drugs before procedures are available. Therefore, there are concerns that the discontinuation of antithrombotic drugs may worsen the disease $\mathrm{e}^{19,20}$. In this retrospective cohort study of patients who received percutaneous interventions for liver diseases, we evaluated the influence of history of antithrombotic drug administration on the incidence rate of intraperitoneal hemorrhage in patients after percutaneous interventions.

\section{Results}

\section{Patient characteristics}

Baseline clinical characteristics of 1025 patients who underwent percutaneous interventions (liver biopsy and treatment of liver tumor, such as RFA and MWA) are shown in Table 1. There were $648(63.2 \%)$ male patients who underwent percutaneous interventions, and the median age of patients was 73.0 years. Antithrombotic drugs were administered to 163 patients (15.9\%) who underwent percutaneous interventions. A total of 138 patients (13.4\%) were treated with monotherapy of antithrombotic drugs and 25 patients $(2.4 \%)$ received combination therapy. The most common antithrombotic therapy was antiplatelet drugs (102 patients [10.0\%] ), and only 47 patients (4.6\%) were treated with anticoagulant drugs. One hundred and sixty patients discontinued these antithrombotic drugs before percutaneous interventions according to the American Association for the Study of Liver Disease (AASLD) guidelines for liver biopsy and the guidelines on the use of liver biopsy from the BSG ${ }^{17,21}$. In 547 patients who received percutaneous treatments, the number of targeted tumors was $1.5 \pm 0.8$, the average maximum tumor size was $1.63 \pm 0.66 \mathrm{~cm}$, and the minimum distance from the liver surface to targeted tumors was $2.03 \pm 1.85 \mathrm{~cm}$. These patients underwent one or two sessions of percutaneous treatment. Clinical background before percutaneous interventions showed serum albumin levels of $3.72 \pm 0.55 \mathrm{~g} / \mathrm{mL}$, totalbilirubin levels of $0.85 \pm 1.09 \mathrm{mg} / \mathrm{mL}$, prothrombin time levels (PT) of $89.5 \pm 15.7 \%$, and Child-Pugh score of $5.59 \pm 0.94$. Intraperitoneal hemorrhage occurred in 9 patients $(0.88 \%)$, and hemoglobin levels decreased by $1.77 \pm 1.02 \mathrm{~g} / \mathrm{dL}$ after these hemorrhages. Although blood transfusion was required in 4 
patients, invasive hemostatic operation was not required in this study. When patients discontinued antithrombotic therapies before interventions, three-point major adverse cardiovascular events (cardiovascular death, nonfatal myocardial infarction, and nonfatal stroke) were not detected in these patients until discharge to home. Twenty-five patients treated with combination therapy discontinued these drugs according to the AASLD guidelines for liver biopsy and guidelines on the use of liver biopsy from the $\mathrm{BSG}^{17,21}$, and there was no intraperitoneal hemorrhage after interventions in these patients. In conclusion, intraperitoneal hemorrhage occurred in only nine patients $(0.88 \%)$, and these adverse events were not severe.

\section{Risk factors of intraperitoneal hemorrhage in patients after percutaneous interventions}

We evaluated which factors were associated with intraperitoneal hemorrhage after percutaneous interventions (Table 2). Baseline clinical characteristics between the patients with intraperitoneal hemorrhage (positive group) and those without (negative group) were compared. The ratio of patients with liver cirrhosis and the etiology of liver disease were not different between the two groups. The number of patients who underwent percutaneous treatments of liver tumor in the positive group was also higher than those in the negative group $(p=0.1271)$. In addition, the number of patients who were administered antithrombotic drugs in the positive group was slightly higher than those in the negative group ( $p=0.1961)$. Regarding clinical parameters, prothrombin time in the positive group was slightly lower than that in the negative group $(p=0.1683)$.

Thereafter, we assessed the clinical characteristics of 9 patients with intraperitoneal hemorrhage after percutaneous interventions (Table 3). Seven patients underwent percutaneous treatments (RFA or MWA). Intraperitoneal hemorrhage was observed in the treatment of tumors of $2 \mathrm{~cm}$ or less and was also observed in those with a distance of $4 \mathrm{~cm}$ or more from the liver surface. In four patients, no abnormality of hematological parameters with disturbance of thrombolysis or coagulation was detected. Furthermore, three patients were administered antithrombotic drugs, and all of them responded according to the guidelines for antithrombin drugs.

In Table 4, we examined the risk factors for intraperitoneal hemorrhage in patients who underwent percutaneous treatments (RFA and MWA). There were no differences in age, sex, the ratio of cirrhosis, and etiology. The proportion of patients administered antithrombotic drugs was higher in the positive group. However, no significant difference was observed $(p=0.1492)$. The Serum PT level was slightly lower in the positive group $(77.57 \pm 19.92 \%)$ than in the negative group ( $86.34 \pm 14.26 \%)$. Platelet count, serum albumin, and total bilirubin levels did not differ between the groups. Then, we compared tumor factors in the two groups. The number of targeted tumors, maximum size of targeted tumors, and distance from the liver surface to targeted tumors were almost the same between the two groups. These results suggest that percutaneous treatment may be associated with intraperitoneal hemorrhage after treatment in patients with liver diseases. 


\section{Influence of antithrombotic drugs on intraperitoneal hemorrhage in patients who underwent percutaneous interventions}

The influence of antithrombotic drug administration in patients who underwent percutaneous intervention was evaluated. We divided the patients into patients with and without antithrombotic drugs. Because there were some confounding factors in the clinical background in the two groups of patients, we performed propensity score matching for some factors between these two groups of patients (Table 5). A total of 163 patients were matched in the two groups, and most of them in patients treated with antithrombotic drugs and then discontinued these drugs according to the guidelines before percutaneous interventions. There was no difference in liver function and hematological parameters between the two groups. Three patients treated with antithrombotic drugs experienced intraperitoneal hemorrhage after interventions. However, this adverse event was not detected in patients without antithrombotic drugs ( $p=$ 0.0407). Based on these results, the administration of antithrombotic drugs was a risk factor for intraperitoneal hemorrhage in patients who underwent percutaneous interventions.

\section{Discussion}

In this study, we performed interventions according to the guidelines for liver biopsy ${ }^{17}$. According to these guidelines, discontinuation of antithrombotic therapy is recommended in patients before biopsy. However, the evidence is weak. To the best of our knowledge, this is the first study to indicate that administration of antithrombotic drugs is a risk factor for intraperitoneal hemorrhage in patients who underwent percutaneous interventions for liver diseases. In this study, intraperitoneal hemorrhage occurred in 9 patients who underwent percutaneous interventions (0.91\%). In addition, 7 patients had hemorrhage after interventions (1.28\%). It has been reported that hemorrhage was detected in $0.4 \%-0.5 \%$ of patients who underwent abdominal interventions. However, in these reports, hemorrhage was defined as the requirement for blood transfusion after interventions or reducing serum hemoglobin by more than $3 \mathrm{~g} / \mathrm{dL}$ before interventions. In this study, intraperitoneal hemorrhage was defined as that was diagnosed by CT until 7 days after interventions. The patients with intraperitoneal hemorrhage in our study included even milder hemorrhage than in other studies, suggesting an increase in the frequency of these complications. In all 9 patients with intraperitoneal hemorrhage, a reduction in serum hemoglobin level by $3 \mathrm{~g} / \mathrm{dL}$ or more was not observed and there were no abdominal symptoms. In these cases, 5 patients were conservatively followed up with hemostatic agents alone, and 4 patients required blood transfusions. They extended hospital stays by an average of $7.57 \pm 3.82$ days. However, events in all these patients were not fatal. It has been reported that the posterior intercostal artery could be injured by these interventions ${ }^{22}$. There are some reports that hemorrhage could not be controlled by blood transfusion and patients died after these interventions ${ }^{10,23}$. Therefore, these interventions should be performed carefully.

In this study, the patients who underwent RFA or MWA had more adverse events of intraperitoneal hemorrhage than those who underwent liver biopsy. The number of punctures per intervention was higher than that per liver biopsy. At the time of liver biopsy, we performed a single puncture per one intervention. On the other hand, at the time of RFA or MWA, we often treated multiple tumors in the liver. Therefore, 
multiple punctures were required for these treatments. The difference in frequency of hemorrhage between interventions may be due to the number of punctures.

It has been reported that localization of targeted tumors is one of the risk factors for intraperitoneal hemorrhage in patients with liver tumors after percutaneous treatments ${ }^{24}$. A high frequency of hemorrhage occurred in cases where tumors existed near the liver surface ${ }^{7}$. In this study, the distance from the targeted tumors to the liver surface was compared. However, no significant difference was observed in patients with or without hemorrhage. There were two cases of hemorrhage in patients with tumors $\geq 4 \mathrm{~cm}$ from the liver surface. Moreover, in both cases, these patients were administered antithrombotic drugs that were withdrawn according to the guidelines. The distance from the liver surface was a risk for damaging hepatic arteries or portal veins in interventions, and antithrombotic drugs may have been difficult to stop bleeding.

There were 3 patients in whom liver biopsy was performed while administered aspirin and no hemorrhage was observed in all patients after liver biopsy. Regarding aspirin, as stated in the BSG guideline about liver biopsy published in 2020, if it cannot be discontinued for various reasons, it may be acceptable to perform under administration ${ }^{17}$. According to the guidelines for gastroenterological endoscopy in patients undergoing antithrombotic treatment from the Japanese Gastroenterological Endoscopy Society, thromboembolic complications due to warfarin withdrawal in procedures occurred in 12 stroke of 1137 cases. It has been reported that those who are 80 years old, a history of stroke, and hyperlipidemia were risk factors for stroke due to warfarin withdrawal ${ }^{25,26}$. In this study, the antithrombotic therapies were discontinued before the intervention, three-point major adverse cardiovascular events did not occur in these patients ${ }^{27}$. This may be related to the inherent abnormalities of hematological parameters, with disturbance of both thrombolysis and coagulation in patients with chronic liver disease.

This study has several limitations. It has all limitations inherent to retrospective studies: prone to selection bias and subject to confounding. However, we performed propensity score matching for some factors between these the two groups of patients to avoid the effect of confounding variables on the results. The small sample size is another limitation to the study that could have reduced the power of the study and increased the margin of error.

In conclusions, the administration of antithrombotic drugs was a risk factor for intraperitoneal hemorrhage in patients who underwent percutaneous interventions. It is also necessary to deeply consider whether these interventions are needed in these patients. In addition, these interventions should be performed carefully.

\section{Methods}

\section{Ethics statement}


This retrospective study was approved by the Institutional Review Board of Osaka Medical College (IRB approval number: 2020-070). Informed consent was obtained in the form of opt-out, and patients who rejected them were excluded from the study. Ethical approval was obtained from the Ethical and Scientific Committee of the Osaka Medical College.

\section{Accordance and guideline}

All procedures performed in this study were in accordance with the ethical standards of the institution and ethical guideline for medical and human subject in Japan and with the 1964 Helsinki declaration and its later amendments.

\section{Study design and human participants}

At Osaka Medical College Hospital, 1025 patients who underwent percutaneous interventions for liver diseases (liver biopsy and percutaneous ablation such as RFA and MWA) from April 2015 to March 2020 were enrolled in this study. All interventions were performed under an ultrasound-guided approach using XALIO ultrasound system or APLIO i800 (Canon Medical Systems Corporation, Tochigi, Japan). Two hundred and sixty-three patients underwent non-targeted liver biopsy and 181 patients underwent targeted biopsy for liver tumors. A total of 547 patients who were diagnosed with liver tumors (522 hepatocellular carcinoma and 25 metastatic other cancers) underwent percutaneous interventions (RFA or MWA). Four hundred and ninety-nine patients underwent RFA and 57 patients underwent MWA. We retrospectively collected the following patient data on their clinical backgrounds: age, sex, etiology of liver disease, administration of antithrombotic drugs, laboratory data, and information about tumors (tumor size, number of tumors, and distance from the liver surface to tumor). We used a 14-G side-cutting needle for liver biopsy and a 21-G needle for targeted biopsy for liver tumors. Regarding percutaneous interventions for treatment, we used 17-G needles (cool-tip ${ }^{\text {TM }}$ RF needle) for RFA and 14-G needles (Thermosphere Technology with Emprint ${ }^{\text {TM }}$ Long Percutaneous Antenna) for MWA. When patients administered antithrombotic drugs were scheduled to undergo percutaneous interventions, antiplatelet drugs were paused 7days before interventions. In addition, warfarin was discontinued 5 days before intervention with point-of-care testing before the intervention to ensure adequate reversal. Only 28 patients who were administered warfarin were switched from warfarin to low-molecular-weight heparin via subcutaneous injection, and this injection was discontinued 12 hours before interventions. Intraperitoneal hemorrhage after percutaneous interventions was detected by abdominal CT until seven days after interventions.

\section{Statistical analysis}

Clinical laboratory values were not normally distributed. Therefore, the Mann-Whitney $U$ test was used to analyze proportional scales. Fisher's exact test was used to analyze nominal scales. All recorded $p$ values were two-sided, and a value of $p<0.05$ was considered statistically significant. All analyses were performed using JMP software, version 13 (SAS Institute Inc., Cary, NC, USA). In the analysis of the prevalence of intraperitoneal hemorrhage in patients who were administered antithrombotic drugs and 
without, propensity score matching was used by using variables of the entire logistic regression model. In this analysis, we performed the matching for some factors (age, sex, liver cirrhosis, and percutaneous treatments).

\section{Declarations}

\section{Acknowledgements}

We would like to thank Editage (www.editage.com) for English language editing.

\section{Author contributions}

Conception and design of the study: Akira Asai and Keisuke Yokohama

Acquisition of data: Akira Asai, Keisuke Yokohama, Hideko Ohama and Yusuke Tsuchimoto

Statistical analysis: Keisuke Yokohama and Akira Asai

Interpretation of data and drafting manuscript: Akira Asai and Keisuke Yokohama

Critical revision and study supervision: Akira Asai, Shinya Fukunishi and Kazuhide Higuchi

\section{Additional information}

Competing Interests: The authors declare no competing interests.

Correspondence and requests should be addressed to A.A.

\section{References}

1. Chalasani, N. et al. The diagnosis and management of nonalcoholic fatty liver disease: Practice guidance from the American Association for the Study of Liver Diseases. Hepatology. 67, 328-357 https://doi.org/10.1002/hep.29367 (2018).

2. EASL-EASD-EASO Clinical Practice Guidelines for the management of non-alcoholic fatty liver disease. Journal of hepatology. 64, 1388-1402 (2016).

3. Fang, Y. et al. Comparison of long-term effectiveness and complications of radiofrequency ablation with hepatectomy for small hepatocellular carcinoma. J Gastroenterol Hepatol. 29, 193-200 https://doi.org/10.1111/jgh.12441 (2014).

4. Shaw, C. \& Shamimi-Noori, S. Ultrasound and CT-directed liver biopsy. Clin Liver Dis (Hoboken). 4, 124-127 https://doi.org/10.1002/cld.437 (2014).

5. The global. regional, and national burden of cirrhosis by cause in 195 countries and territories, 1990-2017: a systematic analysis for the Global Burden of Disease Study 2017. Lancet Gastroenterol Hepatol. 5, 245-266 https://doi.org/10.1016/s2468-1253(19)30349-8 (2020). 
6. Fitzmaurice, C. et al. Global, Regional, and National Cancer Incidence, Mortality, Years of Life Lost, Years Lived With Disability, and Disability-Adjusted Life-Years for 29 Cancer Groups, 1990 to 2017: A Systematic Analysis for the Global Burden of Disease Study. JAMA Oncol. 5, 1749-1768 https://doi.org/10.1001/jamaoncol.2019.2996 (2019).

7. Livraghi, T. et al. Treatment of focal liver tumors with percutaneous radio-frequency ablation: complications encountered in a multicenter study. Radiology. 226, 441-451 https://doi.org/10.1148/radiol.2262012198 (2003).

8. Maeda, M. et al. Complications after Radiofrequency Ablation for Hepatocellular Carcinoma: A Multicenter Study Involving 9,411 Japanese Patients. Liver Cancer. 9, 50-62 https://doi.org/10.1159/000502744 (2020).

9. Park, J. G. et al. Early complications after percutaneous radiofrequency ablation for hepatocellular carcinoma: an analysis of 1,843 ablations in 1,211 patients in a single centre: experience over 10 years. Clin Radiol. 72, 692 https://doi.org/.e699-692.e615 (2017).

10. Ding, J. et al. Complications of thermal ablation of hepatic tumours: comparison of radiofrequency and microwave ablative techniques. Clin Radiol. 68, 608-615 https://doi.org/10.1016/j.crad.2012.12.008 (2013).

11. Dmytriw, A. A., Rivers-Bowerman, M. D. \& Woodley-Cook, J. Percutaneous transcatheter embolisation of active haemorrhage following radiofrequency ablation of hepatocellular carcinoma. BMJ Case Rep 2016, doi:10.1136/bcr-2016-215466 (2016).

12. Lisman, T., Kamphuisen, P. W., Northup, P. G. \& Porte, R. J. Established and new-generation antithrombotic drugs in patients with cirrhosis - possibilities and caveats. J Hepatol. 59, 358-366 https://doi.org/10.1016/j.jhep.2013.03.027 (2013).

13. Beste, L. A. et al. Trends in burden of cirrhosis and hepatocellular carcinoma by underlying liver disease in US veterans, 2001-2013. Gastroenterology. 149, 1471-1482 e1475; quiz e1417-1478 https://doi.org/10.1053/j.gastro.2015.07.056 (2015).

14. Younossi, Z. M. Non-alcoholic fatty liver disease - A global public health perspective. J Hepatol. 70 , 531-544 https://doi.org/10.1016/j.jhep.2018.10.033 (2019).

15. Younossi, Z. M. et al. Global epidemiology of nonalcoholic fatty liver disease-Meta-analytic assessment of prevalence, incidence, and outcomes. Hepatology. 64, 73-84 https://doi.org/10.1002/hep.28431 (2016).

16. Lonardo, A., Nascimbeni, F., Mantovani, A. \& Targher, G. Hypertension, diabetes, atherosclerosis and NASH: Cause or consequence? J Hepatol. 68, 335-352 https://doi.org/10.1016/j.jhep.2017.09.021 (2018).

17. Neuberger, J. et al. Guidelines on the use of liver biopsy in clinical practice from the British Society of Gastroenterology, the Royal College of Radiologists and the Royal College of Pathology. Gut. 69, 1382-1403 https://doi.org/10.1136/gutjnl-2020-321299 (2020).

18. Keeling, D., Tait, R. C. \& Watson, H. Peri-operative management of anticoagulation and antiplatelet therapy. Br J Haematol. 175, 602-613 https://doi.org/10.1111/bjh.14344 (2016). 
19. Blacker, D. J., Wijdicks, E. F. \& McClelland, R. L. Stroke risk in anticoagulated patients with atrial fibrillation undergoing endoscopy. Neurology. 61, 964-968 https://doi.org/10.1212/01.wnl.0000086817.54076.eb (2003).

20. Sibon, I. \& Orgogozo, J. M. Antiplatelet drug discontinuation is a risk factor for ischemic stroke. Neurology. 62, 1187-1189 https://doi.org/10.1212/01.wnl.0000118288.04483.02 (2004).

21. Rockey, D. C., Caldwell, S. H., Goodman, Z. D., Nelson, R. C. \& Smith, A. D. Liver biopsy. Hepatology (Baltim., Md.). 49, 1017-1044 https://doi.org/10.1002/hep.22742 (2009).

22. Vajtai, Z. \& Roy, N. Intercostal artery pseudoaneurysm after ultrasound-guided liver biopsy: a case report and review of the literature. Ultrasound Q. 31, 63-65 https://doi.org/10.1097/ruq.0000000000000074 (2015).

23. Giorgio, A. et al. Percutaneous radiofrequency ablation of hepatocellular carcinoma in cirrhosis: analysis of complications in a single centre over 20 years. Br J Radiol. 90, 20160804 https://doi.org/10.1259/bjr.20160804 (2017).

24. Teratani, T. et al. Radiofrequency ablation for hepatocellular carcinoma in so-called high-risk locations. Hepatology. 43, 1101-1108 https://doi.org/10.1002/hep.21164 (2006).

25. Fujimoto, K. et al. Guidelines for gastroenterological endoscopy in patients undergoing antithrombotic treatment. Dig Endosc. 26, 1-14 https://doi.org/10.1111/den.12183 (2014).

26. Kato, M. et al. Guidelines for Gastroenterological Endoscopy in Patients Undergoing Antithrombotic Treatment: 2017 Appendix on Anticoagulants Including Direct Oral Anticoagulants. Dig Endosc. 30, 433-440 https://doi.org/10.1111/den.13184 (2018).

27. Gage, B. F. et al. Validation of clinical classification schemes for predicting stroke: results from the National Registry of Atrial Fibrillation. Jama. 285, 2864-2870 https://doi.org/10.1001/jama.285.22.2864 (2001).

\section{Tables}

Due to technical limitations, table $1,2,3,4,5$ is only available as a download in the Supplemental Files section.

\section{Supplementary Files}

This is a list of supplementary files associated with this preprint. Click to download.

- Tables.docx 